\title{
Nijenhuis infinity and contractible differential graded manifolds
}

\author{
S. A. Merkulov
}

\begin{abstract}
We find a minimal differential graded ( $\mathrm{dg}$ ) operad whose generic representations in $\mathbb{R}^{n}$ are in one-to-one correspondence with formal germs of those endomorphisms of the tangent bundle to $\mathbb{R}^{n}$ which satisfy the Nijenhuis integrability condition. This operad is of a surprisingly simple origin: it is the cobar construction on the quadratic operad of homologically trivial dg Lie algebras. As a by-product we obtain a strong-homotopy generalization of this geometric structure and show its homotopy equivalence to the structure of contractible dg manifolds.
\end{abstract}

\section{Introduction}

Extended deformation theory of a mathematical structure $\mathfrak{X}$ begins with the construction of a 'controlling' algebra, $\mathfrak{g}$, over an operad $\mathcal{D}$ ef. The formal smooth extended moduli space of deformations of $\mathfrak{X}$ is then a homotopy class of minimal algebras over its cofibrant resolution, $\mathcal{D} e f_{\infty}$, canonically induced on the cohomology of $\mathfrak{g}$. The operad $\mathcal{D}$ ef often (if not always) contains the suboperad of Lie algebras which in turn explains why a part of the data induced on the extended moduli space can also be effectively described with the help of the classical idea of the deformation functor on differential graded (dg) Artinian rings. For example, if $\mathfrak{X}$ is either the structure of an associative algebra on a vector space $V$, or a symplectic/complex structure on a manifold $M$, then the appropriate $\mathcal{D} e f_{\infty}$ is the minimal resolution of the operad of Gerstenhaber algebras, a fact from which we can, for example, immediately infer that the associated extended moduli space (modeled as a formal supermanifold on the cohomology spaces $\operatorname{Hoch}^{\bullet}(A, A), H_{\text {deRham }}^{\bullet}(M)$, and $H_{\text {Dolbeault }}^{\bullet}\left(M, \wedge T_{M}\right)$, respectively, in the three cases just mentioned) has canonically induced a Hertling-Manin ${ }_{\infty}$ geometric structure [HM99, Mer04a].

This machinery does not explain, however, what happens to the structure $\mathfrak{X}$ itself upon generic extended deformation, that is, upon deformation in a direction other than in $\operatorname{Hoch}^{2}(A, A)$, $H_{\text {deRham }}^{2}(M)$ or $H_{\text {Dolbeault }}^{1}\left(M, T_{M}\right)$, respectively. Thanks to Stasheff [Sta63] we know what happens to the associative algebra structure under generic deformations: it gets transformed into the $A_{\infty}$-structure. Remarkably enough, the latter can also be understood in terms of minimal resolutions of operads! This fact encourages our present attempt to understand some classical geometric structures from the operadic perspective. The hope is that the theory of (di)operads can provide us with an insight into what might be called geometry ${ }_{\infty}$, the typical fibre of a yet unknown 'universal curve' over a generic point of the extended moduli space.

In [Mer04b] we presented a dioperadic profile of Poisson geometry. In this paper we attempt to create an operadic profile of one of the key equations defining complex structure, the Nijenhuis integrability condition. After some preparations in $\S \S 2-4$, we show in the final section, $\S 5$,

Received 18 March 2004, accepted in final form 16 November 2004, published online 1 September 2005.

2000 Mathematics Subject Classification 18G20, 53B99.

Keywords: operads, resolutions, Nijenhuis integrability.

This journal is (C) Foundation Compositio Mathematica 2005. 


\section{NiJENHUIS INFINITY AND CONTRACTIBLE DIFFERENTIAL GRADED MANIFOLDS}

a surprisingly simple minimal dg operad $N_{\infty}$ whose generic representations in $\mathbb{R}^{n}$ are in one-to-one correspondence with formal germs of those endomorphisms of the tangent bundle, $J: T_{\mathbb{R}^{n}} \rightarrow T_{\mathbb{R}^{n}}$, which satisfy the Nijenhuis integrability condition, $N_{J}=0$. Generic representations of $N_{\infty}$ in graded vector spaces provide us then with the strong-homotopy generalization of this geometric structure.

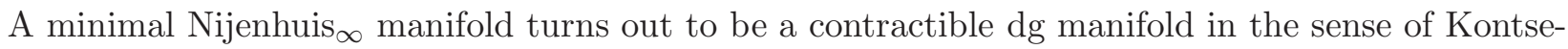
vich [Kon03].

\section{Strongly-homotopy Nijenhuis?}

Let $M$ be a smooth manifold and $J: T_{M} \rightarrow T_{M}$ an endomorphism of the tangent bundle. Nijenhuis [Nij55] used these data to construct a tensor,

$$
\begin{array}{cccc}
N_{J}: & \wedge^{2} T_{M} & \longrightarrow & T_{M} \\
& X \otimes Y & \longrightarrow & N_{J}(X, Y)
\end{array}
$$

where

$$
N_{J}(X, Y):=[J X, J Y]+J^{2}[X, Y]-J[X, J Y]-J[J X, Y] .
$$

By the Newlander-Nirenberg theorem, its vanishing for an almost complex structure $J$ is a necessary and sufficient condition for the latter to be a complex structure. If $J: T_{M} \rightarrow T_{M}$ is a fiberwise diagonalizable endomorphism with all eigenvalues real and of constant multiplicity, then eigenspaces of $J$ are integrable if and only if $N_{J}=0$ [Mic87].

A Nijenhuis geometric structure on $M$ is defined to be an endomorphism $J: T_{M} \rightarrow T_{M}$ satisfying the condition $N_{J}=0$. Complex structures are, probably, the most important examples of Nijenhuis structures.

At present we have good experience in obtaining strongly-homotopy generalizations of classical algebraic structures: the major working tool is the construction of minimal dg resolutions of the operads governing the classical structures (see, e.g., [GJ94, GK94, MSS02]). Can we apply this technique to get, say, a Nijenhuis $\infty_{\infty}$ geometric structure? For this purpose let us first try to translate geometry into algebra, and choose a local coordinate system, $\left\{t^{\alpha}\right\}_{1 \leqslant \alpha \leqslant \operatorname{dim} M}$, at a point $*$ in $M$, that is a local isomorphism of germs of smooth manifolds $\left(V=\mathbb{R}^{n}, 0\right) \rightarrow(M, *)$. The germ at $*$ of any analytic endomorphism $J: T_{M} \rightarrow T_{M}$ is then represented explicitly as

$$
\begin{aligned}
J & =\sum_{\alpha, \beta} J_{\beta}^{\alpha}(t) d t^{\beta} \otimes \frac{\partial}{\partial t^{\alpha}}, \\
J_{\beta}^{\alpha}(t) & =\sum_{k \geqslant 0} \sum_{\gamma_{1}, \ldots, \gamma_{k}} J_{\beta \gamma_{1} \ldots \gamma_{k}}^{\alpha} t^{\gamma_{1}} \ldots t^{\gamma_{k}},
\end{aligned}
$$

i.e. as an infinite collection of 'algebraic operations', $\left\{J_{k}: \otimes^{k+1} V \rightarrow V\right\}_{k \geqslant 0}$. The Nijenhuis integrability constraint then translates into an infinite sequence of quadratic relations between these operations. The experience with algebra, if understood straightforwardly, suggests the following scenario: (1) consider an operad $\mathcal{N}$ which governs these algebraic operations and relations; (2) attempt to construct its cofibrant resolution $\mathcal{N}_{\infty} ;(3)$ study generic representations of the latter in $\mathrm{dg}$ vector spaces; in the particular cases of vector spaces concentrated in degree zero we are guaranteed to get all the operations $J_{k}$ satisfying the required Nijenhuis integrability constraint (plus something else).

However, this scenario is doomed to failure: the input operad $\mathcal{N}$ is already so monstrously large that it is technically impossible (and repelling) to go for something yet larger.

So let us try instead to go for something much smaller, and investigate a theoretical possibility that the Nijenhuis integrability condition is already 'minimally resolved' in the sense that there exists 


\section{S. A. Merkulov}

a minimal dg operad $N_{\infty}$ whose representations in spaces concentrated in degree zero are precisely the collections $\left\{J_{k}: \otimes^{k+1} V \rightarrow V\right\}_{k \geqslant 0}$ satisfying the required quadratic constraints. Instead of steps (1) and (2) above we now face a problem of reconstructing the full minimal dg operad $N_{\infty}$ from just its degree zero bit corresponding to $\mathcal{N}$.

If the guess in the preceding paragraph is correct, then some operation(s) in the family $\left\{J_{k}\right.$ : $\left.\otimes^{k+1} V \rightarrow V\right\}_{k \geqslant 0}$ is (are) fundamental while all the others are just higher homotopies of the fundamental one(s). As the Nijenhuis integrability condition is a system of first-order non-linear partial differential equations we suggest the linear in $t^{a}$ bit, $J_{1}: \otimes^{2} V \rightarrow V$, of the endomorphism tensor $J$ for the role of the fundamental operation, and immediately observe the following.

Lemma 2.1. There is a one-to-one correspondence,

$$
\left\{\begin{array}{c}
\text { Nijenhuis structures linear in } t^{a} \\
J: T_{V} \rightarrow T_{V}
\end{array}\right\} \leftrightarrow\left\{\begin{array}{c}
\text { pre-Lie algebra } \\
\text { structures on } V
\end{array}\right\}
$$

Thus pre-Lie algebras are going to play an important role in the story.

\subsection{Reminder on pre-Lie algebras}

A pre-Lie algebra is a vector space together with a binary operation, $\circ: V^{\otimes 2} \rightarrow V$, satisfying the condition

$$
(a \circ b) \circ c-a \circ(b \circ c)-(-1)^{|b||c|}(a \circ c) \circ b+(-1)^{|b||c|} a \circ(c \circ b)=0
$$

for any $a, b, c \in V$.

A pre-Lie algebra $V$ is naturally a Lie algebra with the bracket,

$$
[a, b]:=a \circ b-(-1)^{|a||b|} b \circ a .
$$

The operad of pre-Lie algebras is Koszul [CL01] and its minimal dg resolution can be explicitly described using the technique of Ginzburg and Kapranov [GK94].

Remark 2.3. Pre-Lie ${ }_{\infty}$ algebra structures will eventually form a (small) part of the Nijenhuis ${ }_{\infty}$ structure. It turns out that to get the full picture of Nijenhuis $_{\infty}$ one has to add to the pre-Lie operation $\circ$ at least one more algebraic operation, [•], the Lie bracket operation in degree one. Such an extension is more than expected: along the way from geometry to algebra in $\S 1$ we fixed a flat structure at $(M, *)$, an ugly thing to do from the geometric viewpoint. If everything is done

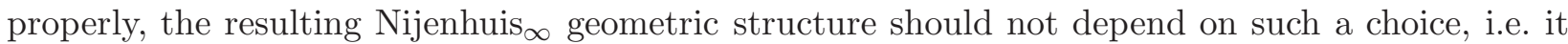
must be tensorial. Thus we must add an ingredient to our algebraic input whose homotopy theory contains the group of general coordinate transformations as homotopy equivalences. Moreover, Lie algebras in degree one just have this property: a minimal Lie ${ }_{\infty}$ algebra structure is the same as a homological vector field on a smooth formal manifold [Kon03]; generic diffeomorphisms of the latter generate homotopy equivalence among the former. Thus a Lie algebra structure in degree one is expected to be present in the (di)operadic profile of any diffeomorphism covariant geometric structure, cf. [Mer04b].

\section{Operad of pre-Lie ${ }^{2}$ algebras}

Here is a required graded extension of the notion of pre-Lie algebra.

Definition 3.1. A pre- $\mathrm{Lie}^{2}$ algebra is a graded vector space $V$ together with two binary operations,

$$
\begin{aligned}
& \circ: V \otimes V \longrightarrow V \quad[\bullet]: \odot^{2} V \quad \longrightarrow \quad V[1] \\
& a \otimes b \quad \longrightarrow a \circ b, \quad a \otimes b \longrightarrow(-1)^{|b|}[a \bullet b]
\end{aligned}
$$

such that: 


\section{NIJENHUIS INFINITY AND CONTRACTIBLE DIFFERENTIAL GRADED MANIFOLDS}

(i) the data $(V, \circ)$ makes $V$ into a pre-Lie algebra;

(ii) the data $(V,[\bullet])$ makes $V[-1]$ into a Lie algebra; equivalently, the odd Jacobi identity,

$$
[[a \bullet b] \bullet c]=[a \bullet[b \bullet c]]+(-1)^{|b||a|+|b|+|a|}[b \bullet[a \bullet c]],
$$

holds for any $a, b, c \in V$;

(iii) a 'compatibility' identity,

$$
\begin{aligned}
{[a \bullet b] \circ c+(-1)^{|b|} a \circ[b \bullet c] } & +(-1)^{|b||a|+|b|} b \circ[a \bullet c] \\
& =(-1)^{|b||c|+|c|}[(a \circ c) \bullet b]+(-1)^{(|a|+1)(|b|+|c|)+|a|}[(b \circ c) \bullet a]
\end{aligned}
$$

holds for any $a, b, c \in V$.

The last condition (iii) should be explained.

3.1.1 Interpretation $I$. Recall that if $(\mathfrak{g},[]$,$) is a Lie algebra and M$ is a right Lie module over $(\mathfrak{g},[]$,$) , then the Chevalley-Eilenberg construction makes M \otimes \wedge \bullet \mathfrak{g}$ into a complex (computing $\left.H_{\bullet}(\mathfrak{g}, M)\right)$.

Similarly, if $(\mathfrak{g},[\bullet])$ is an odd Lie algebra (i.e. [•] satisfies the odd Jacobi identity as in (ii) above), and $M$ is a module over $(\mathfrak{g},[\bullet])$, then the same Chevalley-Eilenberg construction now makes the vector space $\odot \mathfrak{g} \otimes M$ into a complex.

Let $V$ be a graded vector space equipped with two operations $\circ$ and [ $\bullet$ ] satisfying conditions (i) and (ii) in Definition 3.1. Then the vector space $\odot^{\bullet} V \otimes \wedge^{\bullet} V$ has two Chevalley-Eilenberg differentials:

(a) one Chevalley-Eilenberg differential, $d_{\circ}$, comes from the even Lie algebra structure on $V$ (as in $\S 2.2)$ and the right $(V,[]$,$) -module structure on \odot{ }^{\bullet} V$ generated by right o-multiplication;

(b) the other Chevalley-Eilenberg differential, $d_{\bullet}$, comes from the odd Lie algebra structure, $[\bullet]$, on $V$ and the $(V,[\bullet])$-module structure on $\wedge^{\bullet} V$ generated by the adjoint [•]-action.

The condition (iii) in Definition 3.1 is then equivalent to commutativity of the two ChevalleyEilenberg differentials, $d_{\circ}$ and $d_{\bullet}$.

3.1.2 Interpretation $I I$. If $V$ is a vector space, then $V \oplus V[-1]$ is naturally a complex with trivial cohomology. If we write elements of $V \oplus V[-1]$ as $a+\Pi b$, where $a, b \in V$ and $\Pi$ is a formal symbol of degree one, then the differential is given by

$$
d(a+\Pi b)=0+\Pi a .
$$

It is straightforward to check that a pair of linear maps, $\circ: \otimes^{2} V \rightarrow V$ and $[\bullet]: \odot{ }^{2} V \rightarrow V[1]$, makes $V$ into a pre-Lie ${ }^{2}$-algebra if and only if the brackets on $V \oplus V[-1]$,

$$
\begin{aligned}
{[a, b] } & :=a \circ b-(-1)^{|a||b|} b \circ a, \\
{[\Pi a, b] } & :=-(-1)^{|a|}[a \bullet b]+\Pi a \circ b, \\
{[\Pi a, \Pi b] } & :=\Pi[a \bullet b],
\end{aligned}
$$

make the complex $(V \oplus V[-1], d)$ into a dg Lie algebra.

Example 3.1.3. Let $W$ be a vector space and Free $_{\text {Lie }}(W \oplus W[-1])$ the free Lie algebra on the vector space $W \oplus W[-1]$. Consider two differentials,

$$
\begin{aligned}
& d: W \oplus W[-1] \quad \longrightarrow W \oplus W[-1] \quad q: W \oplus W[-1] \quad \longrightarrow W \oplus W[-1] \\
& a+\Pi b \quad \longrightarrow \quad 0+\Pi a, \quad \longrightarrow \quad b+\Pi b,
\end{aligned}
$$




\section{S. A. Merkulov}

and extend them to Free $_{\text {Lie }}(W \oplus W[-1])$ as derivations of the Lie bracket. For any Lie word $A$ of length $\lambda_{A}$ one has $(d q+q d) A=\lambda_{A} A$ so that after rescaling $q \rightarrow Q:=(\lambda(\ldots))^{-1} q$ we get eventually two differentials on Free $_{\text {Lie }}(W \oplus W[-1])$ which satisfy

$$
d Q+Q d=\mathrm{Id}
$$

Hence Free $_{\text {Lie }}(W \oplus W[-1])$ splits canonically into the direct sum, $V \oplus V[-1]$, where $V=\operatorname{Im} Q$. Using interpretation II in $\S 3.1 .2$ it is now easy to check that the vector space $V$ is naturally a pre-Lie ${ }^{2}$ algebra with

$$
\begin{aligned}
Q a \circ Q b & :=Q[d Q a, Q b], \\
{[Q a \bullet Q b] } & :=Q[d Q a, d Q b],
\end{aligned}
$$

for any $a, b, c \in$ Free $_{\mathrm{Lie}}(W \oplus W[-1])$. For example, if $w_{1}, w_{2}, w_{3} \in W \subset V$, then one has

$$
\begin{aligned}
w_{1} \circ w_{2} & =\frac{1}{2}\left[w_{1}, w_{2}\right], \\
{\left[w_{1} \bullet w_{2}\right] } & =\frac{1}{2}\left[w_{1}, \Pi w_{2}\right]-(-1)^{\left|w_{1}\right|} \frac{1}{2}\left[\Pi w_{1}, w_{2}\right], \\
w_{1} \circ\left(w_{2} \circ w_{3}\right) & =\frac{1}{6}\left[w_{1},\left[w_{2}, w_{3}\right]\right], \\
\left(w_{1} \circ w_{2}\right) \circ w_{3} & =\frac{1}{3}\left[\left[w_{1}, w_{2}\right], w_{3}\right],
\end{aligned}
$$

and so on.

In fact any Lie algebra with two derivations, $d$ and $Q$, of the Lie bracket satisfying $d^{2}=Q^{2}=0$ and $d Q+Q d=\mathrm{Id}$ gives rise to a pre-Lie ${ }^{2}$ algebra structure on $\operatorname{Im} Q \subset \mathfrak{g}$.

Example 3.1.4. Let $M$ be a smooth manifold and $\Omega_{M}^{\bullet}$ the associated graded commutative de Rham algebra of differential forms. We shall show that derivations of the latter naturally give rise to one more example of a pre-Lie ${ }^{2}$ algebra.

Instead of $M$ it is more suitable to introduce a $(\operatorname{dim} M \mid \operatorname{dim} M)$-dimensional supermanifold $\mathcal{M}:=T_{M}[1]$, the total space of the tangent bundle to $M$ with the degree of a typical fibre shifted by 1 . Then the de Rham algebra $\Omega_{M}^{\bullet}$ is identified with the algebra, $\mathcal{O}_{\mathcal{M}}$, of smooth functions on $\mathcal{M}$, so that the Lie algebra of derivations of $\Omega_{M}^{\bullet}$ is identified with the Lie algebra, $T_{\mathcal{M}}$, of smooth vector fields on $\mathcal{M}$. The de Rham differential becomes a degree one vector field $d$ on $\mathcal{M}$ satisfying $[d, d]=0$.

There is a natural fibration $\pi: \mathcal{M} \rightarrow M$ so that we can define a subset $V \subset T_{\mathcal{M}}$ by the exact sequence,

$$
0 \longrightarrow V \longrightarrow T_{\mathcal{M}} \longrightarrow \pi^{*} T_{M} \longrightarrow 0 \text {. }
$$

This is the space of vertical vector fields on $\mathcal{M}$ and hence it corresponds precisely to the space of those derivations of the de Rham algebra $\Omega_{M}^{\bullet}$ which vanish on functions, $\Omega_{M}^{0}=\mathcal{O}_{M}$, i.e. which are tensorial maps $\Omega_{M}^{1} \rightarrow \Omega_{M}^{\bullet}$. Thus $V$ is canonically isomorphic to the vector space of global sections of the bundle $T_{M} \otimes \Omega_{M}^{\bullet}$ (which we denote by the same symbol as the bundle). Let $i: T_{M} \otimes \Omega_{M}^{\bullet} \rightarrow V$ be that isomorphism. As $V$ is an integrable distribution, it is a Lie subalgebra of $T_{\mathcal{M}}$. Hence the standard Lie algebra structure on $T_{\mathcal{M}}$ induces, upon restriction to $V$ and the isomorphism $i$, a Lie algebra structure, $[,]_{N R}$, on $T_{M} \otimes \Omega_{M}^{\bullet}$ which, in the literature, is often called the NijenhuisRichardson brackets (see, e.g., [Mic87]).

Consider a map,

$$
\begin{array}{ll}
\circ: & T_{M} \otimes \Omega_{M}^{\bullet} \times T_{M} \otimes \Omega_{M}^{\bullet} \longrightarrow T_{M} \otimes \Omega_{M}^{\bullet} \\
& \left.v_{1} \otimes \omega_{1} \times v_{2} \otimes \omega_{2} \longrightarrow v_{2} \otimes\left(\omega_{1} \wedge v_{1}\right\lrcorner \omega_{2}\right) .
\end{array}
$$

It is easy to check that

$$
[a, b]_{N R}=a \circ b-(-1)^{(p+1)(q+1)} b \circ a
$$

for any $a \in T_{M} \otimes \Omega_{M}^{p}$ and $b \in T_{M} \otimes \Omega_{M}^{q}$. 


\section{NiJENHUIS INFINITY AND CONTRACTIBLE DIFFERENTIAL GRADED MANIFOLDS}

Then consider a subspace of $T_{\mathcal{M}}$ consisting of vector fields which commute with the de Rham vector field $d$. It is in fact canonically isomorphic to $V[-1]$ : if $X \in T_{\mathcal{M}}$ satisfies $[d, X]=0$ then $X=[d, i(Y)]$ for some uniquely defined $Y \in T_{M} \otimes \Omega_{M}^{\circ}$. Moreover, this subspace is again a Lie subalgebra of $T_{\mathcal{M}}$. Hence the standard Lie algebra structure on $T_{\mathcal{M}}$ induces on $V \simeq T_{M} \otimes \Omega_{M}^{\bullet}$ the structure, $[\bullet]$, of odd Lie algebra,

$$
\left[\left[d, i\left(X_{1}\right)\right],\left[d, i\left(X_{2}\right)\right]\right]=:\left[d, i\left(\left[X_{1} \bullet X_{2}\right]\right)\right] .
$$

In the literature these brackets [•] are often called the Frölicher-Nijenhuis brackets.

An arbitrary vector field, $X$, on $\mathcal{M}$ can always be decomposed into a sum,

$$
X=i\left(X_{1}\right)+\left[d, i\left(X_{2}\right)\right],
$$

for some uniquely defined $X_{1}, X_{2} \in T_{M} \otimes \Omega_{M}^{\bullet}$. Put another way, $T_{\mathcal{M}}=V \oplus V[-1]$ and, as is not hard to check, the standard Lie bracket on $T_{\mathcal{M}}$ is represented in terms of operations $\circ$ and $[\bullet]$ precisely as in $\S 3.1 .2$.

We conclude that $T_{M} \otimes \Omega_{M}^{\bullet}$ together with the Nijenhuis-Richardson composition $\circ$ and the Frölicher-Nijenhuis brackets $[\bullet]$ is a pre-Lie ${ }^{2}$ algebra.

\subsection{Operads, free operads and trees}

An operad is a pair of collections,

$$
\mathcal{O}=\left(\{\mathcal{O}(n)\}_{n \geqslant 1},\left\{\mathcal{O}_{i}^{n, n^{\prime}}\right\}_{\substack{n, n^{\prime} \geqslant 1 \\ 1 \leqslant i \leqslant n}}\right),
$$

where

(i) $\{\mathcal{O}(n)\}_{n \geqslant 1}$ is an $\mathbb{S}$-module, i.e. a collection of $\mathbb{Z}$-graded vector spaces $\mathcal{O}(n)$ equipped with a linear right action of the permutation group $\mathbb{S}_{n}$;

(ii) $\left\{\mathrm{o}_{i}^{n, n^{\prime}}\right\}_{\substack{n, n^{\prime} \geqslant 1 \\ 1 \leqslant i \leqslant n}}$ is a collection of linear equivariant maps, $\circ_{i}^{n, n^{\prime}}: \mathcal{O}(n) \otimes \mathcal{O}\left(n^{\prime}\right) \longrightarrow \mathcal{O}\left(n+n^{\prime}-1\right)$, satisfying associativity conditions which can be found, e.g., in [MSS02].

A dg operad is an operad $\mathcal{O}$ equipped with a degree one equivariant linear map $d: \mathcal{O}(n) \rightarrow \mathcal{O}(n)$, $\forall n$, satisfying the conditions

$$
\begin{aligned}
d^{2} & =0 \\
d\left(f \circ \circ_{i}^{n, n^{\prime}} f^{\prime}\right) & =(d f) \circ_{i}^{n, n^{\prime}} f^{\prime}+(-1)^{|f|} f \circ_{i}^{n, n^{\prime}} d f^{\prime}, \quad \forall f \in \mathcal{O}(n), f^{\prime} \in \mathcal{O}\left(n^{\prime}\right) .
\end{aligned}
$$

The associated cohomology $\mathbb{S}$-module $H(\mathcal{O}):=\left\{H^{\bullet}(\mathcal{O}(n))\right\}_{n \geqslant 1}$ has an induced operad structure.

An ideal in an operad $\mathcal{O}$ is a collection $I$ of $\mathbb{S}_{n}$-invariant subspaces $\{\mathcal{I}(n) \subset \mathcal{O}(n)\}_{n \geqslant 1}$ such that $f \circ{ }_{i}^{n, n^{\prime}} f^{\prime} \in \mathcal{I}\left(n+n^{\prime}-1\right)$ whenever $f \in \mathcal{I}(n)$ or $f^{\prime} \in \mathcal{I}\left(n^{\prime}\right)$; in particular, $\mathcal{I}$ is a suboperad of $\mathcal{O}$. It is clear that the quotient $\mathbb{S}$-module $\{\mathcal{O}(n) / \mathcal{I}(n)\}_{n \geqslant 1}$ has an induced structure of an operad called the quotient operad.

An operad $\mathcal{O}$ with $\mathcal{O}(1)=0$ is said to be simply connected.

With any graded vector space $V$ one can associate an endomorphism operad,

$$
\mathcal{E}_{V}=\left\{\mathcal{E}_{V}(n):=\operatorname{Hom}\left(V^{\otimes n}, V\right),\left\{0_{i}^{n, n^{\prime}}\right\}_{\substack{n, n^{\prime} \geqslant 1 \\ 1 \leqslant i \leqslant n}}\right\}
$$

where the composition, $f \circ \circ_{i}^{n, n^{\prime}} f^{\prime}$, is just the insertion of the output of $f^{\prime} \in \operatorname{Hom}\left(V^{\otimes n^{\prime}}, V\right)$ into the $i$ th input of $f \in \operatorname{Hom}\left(V^{\otimes n}, V\right)$.

An algebra over an operad $\mathcal{O}$ is, by definition, a $\mathbb{Z}$-graded vector space $V$ together with a morphism of operads $\mathcal{O} \rightarrow \mathcal{E}_{V}$. 


\section{S. A. Merkulov}

With any $\mathbb{S}$-module $\mathcal{E}=\{\mathcal{E}(n)\}_{n \geqslant 1}$ one can associate an operad, Free $(\mathcal{E})$, called the free operad on $\mathcal{E}$. Its construction uses decorated trees which are defined as follows.

Let $n$ be a natural number and let $[n]$ denote the set $\{1,2, \ldots, n\}$. An $[n]$-tree $T$ is, by definition, the data $\left(V_{T}, N_{T}, \phi_{T}\right)$ consisting of:

- a stratified finite set $V_{T}=V_{T}^{i} \sqcup V_{T}^{t}$ whose elements are called vertices; elements of the subset $V_{T}^{i}$ (respectively $V_{T}^{t}$ ) are called internal (respectively tail) vertices;

- a bijection $\phi: V_{T}^{t} \rightarrow[n]$;

- a map $N_{T}: V_{T} \rightarrow V_{T}$ satisfying the conditions: (i) $N_{T}$ has only one fixed point root $_{T}$ which lies in $V_{T}^{i}$ and is called the root vertex, (ii) $N_{T}^{k}(v)=\operatorname{root}_{T}, \forall v \in V_{T}$ and $k \gg 1$, (iii) for all $v \in V_{T}^{i}$ the cardinality, \#v, of the set $N_{T}^{-1}(v)$ is greater than or equal to 1 , while for all $v \in V_{T}^{t}$ one has $\# v=0$.

The number $\# v$ is often called the valency of the vertex $v$; the pairs $\left(v, N_{T}(v)\right)$ are called edges.

Given an $\mathbb{S}$-module $\mathcal{E}=\{\mathcal{E}(n)\}_{n \geqslant 1}$, we can associate to an $[n]$-tree $T$ the vector space

$$
\mathcal{E}(T):=\bigotimes_{v \in V_{T}^{i}} \mathcal{E}(\# v)
$$

Its elements are interpreted as $[n]$-trees whose internal vertices are decorated with elements of $\mathcal{E}$. The permutation group $\mathbb{S}_{n}$ acts on this space via relabelling of the tail vertices (i.e changing $\phi_{T}$ to $\left.\sigma \circ \phi_{T}, \sigma \in \mathbb{S}_{n}\right)$.

Now, as an $\mathbb{S}$-module the free operad $\operatorname{Free}(\mathcal{E})$ is defined as

$$
\operatorname{Free}(\mathcal{E})(n)=\bigoplus_{[[n] \text {-trees } T]} \mathcal{E}(T),
$$

where the summation goes over all isomorphism classes of $[n]$-trees. The composition, say $f \circ \circ_{i}^{n, n^{\prime}} f^{\prime}$, is given by gluing the root vertex of the decorated $[n]$-tree $f \in \operatorname{Free}(\mathcal{E})(n)$ with the $i$-labeled tail vertex of the decorated $\left[n^{\prime}\right]$-tree $f^{\prime}$. The new numeration, $\phi: V_{T}^{t} \rightarrow\left[n+n^{\prime}-1\right]$, of tails is clear.

For an $\mathbb{S}$-module $\mathcal{O}=\{\mathcal{O}(n)\}_{n \geqslant 1}$ we define $\mathcal{O}\{m\}$ to be the $\mathbb{S}$-module given by the tensor product

$$
\mathcal{O}\{m\}(n):=\mathcal{O}(n) \otimes_{k} \operatorname{sgn}_{n}^{\otimes m}[m(1-n)],
$$

where $\operatorname{sgn}_{n}$ is the sign representation of the permutation group $\mathbb{S}_{n}$. If $\mathcal{O}$ is a dg operad, then $\mathcal{O}\{m\}$ is naturally a $\mathrm{dg}$ operad as well: the structure of a $\mathcal{O}\{m\}$-algebra on a dg vector space $V$ is the same as structure of a $\mathcal{O}$-algebra on the shifted dg vector space $V[m]$.

Let $\mathcal{O}=\left\{\mathcal{O}(n), \circ_{i}^{n, n^{\prime}}, d\right\}$ be a simply connected operad with all vector spaces $\mathcal{O}(n)$ being finite dimensional, and let $\mathcal{O}^{*}[-1]$ stand for the $\mathbb{S}$-module $\left\{\mathcal{O}(n)^{*}[-1]\right\}_{n \geqslant 2}$. It was shown in [GK94] that the free operad Free $\left(\mathcal{O}^{*}[-1]\{-1\}\right)$ can be naturally made into a differential operad,

$$
\mathbf{D O}=\left(\operatorname{Free}\left(\mathcal{O}^{*}[-1]\{-1\}\right), d\right),
$$

with the differential $d$ defined by the dualization of the operadic compositions $\circ_{i}^{n, n^{\prime}}$. This construction plays a special role in the theory of so-called quadratic operads, the ones which can be represented as a quotient,

$$
\mathcal{O}=\frac{\operatorname{Free}(\mathcal{E})}{\langle R\rangle},
$$

of the free operad generated by an $\mathbb{S}$-module $\mathcal{E}$ with $\mathcal{E}(n)=0$ for $n \neq 2$ by an ideal generated by an $\mathbb{S}_{3}$-invariant subspace $R$ in $\operatorname{Free}(\mathcal{E})(3)$.

The Koszul dual of a quadratic operad $\mathcal{O}=\operatorname{Free}(\mathcal{E}) /\langle R\rangle$ is, by definition, the quadratic operad $\mathcal{O}^{!}=\operatorname{Free}(\check{\mathcal{E}}) /\left\langle R^{\perp}\right\rangle$, where $\check{\mathcal{E}}$ is the $\mathbb{S}$-module whose only non-vanishing component is $\check{\mathcal{E}}(2)=$ $\mathcal{E}(2)^{*} \otimes \operatorname{sgn}_{2}$ and $R^{\perp}$ is the annihilator of $R$, i.e. the kernel of the natural map Free $(\check{\mathcal{E}})(3) \rightarrow R^{*}$. 


\section{NIJENHUIS INFINITY AND CONTRACTIBLE DIFFERENTIAL GRADED MANIFOLDS}

Applying the cobar construction to the Koszul dual of a quadratic operad $\mathcal{O}$, one gets a cofibrant dg operad $\mathbf{D} \mathcal{O}^{\text {! }}$ together with a canonical map of dg operads [GK94],

$$
\left.(\mathbf{D O})^{!}, d\right) \longrightarrow(\mathcal{O}, 0) \text {. }
$$

If this map happens to be an isomorphism, the operad $\mathcal{O}$ is said to be Koszul and then $\left(\mathbf{D} \mathcal{O}^{!}, d\right)$ provides the minimal dg resolution of $\mathcal{O}$.

For any simply connected operad $\mathcal{O}$ the associated operad $\mathbf{D}\left(\mathcal{O}^{!}\right)$is minimal, i.e. its differential is decomposable. Thus the cobar construction, that is the functor

$$
\mathcal{O} \rightarrow \mathcal{O}_{\infty}:=\left(\mathbf{D} \mathcal{O}^{!}, d\right)
$$

associates with any quadratic operad a minimal dg operad whose algebras are strongly homotopic (i.e. are transferable via quasi-isomorphisms of complexes) and whose homology operad covers $\mathcal{O}$.

\subsection{Operad of pre-Lie ${ }^{2}$ algebras}

Let us denote this operad by $\mathcal{P}$. Clearly, it is quadratic, $\mathcal{P}=\operatorname{Free}(\mathcal{E}) /\langle R\rangle$, with $\mathcal{E}(2):=k\left[\mathbb{S}_{2}\right][0] \oplus$ $\mathbf{1}_{2}[-1]$ and the relations in Definition 3.1(i)-(iii). Here and elsewhere, $\mathbf{1}_{n}$ (respectively, $k\left[\mathbb{S}_{n}\right]$ ) stands for the one-dimensional trivial (respectively, $n$ !-dimensional regular) representation of $\mathbb{S}_{n}$.

As the free operad $\operatorname{Free}(\mathcal{E})$ is a construction on binary trees, it will be useful to have corolla type notation for the generators of $\mathcal{E}(2)$ : we shall represent the two basis vectors, (1)(2) and (12), of the summand $k\left[\mathbb{S}_{2}\right][0]$ by the labeled planar corollas of degree zero,

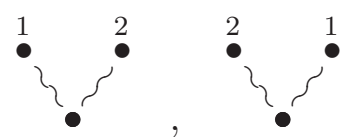

while the basis vector of the summand $\mathbf{1}_{2}[-1]$ by the unique space corolla of degree one,

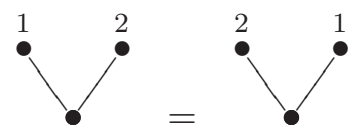

Then the ideal $\langle R\rangle$ is generated by the following elements of Free $(\mathcal{E})(3)$,
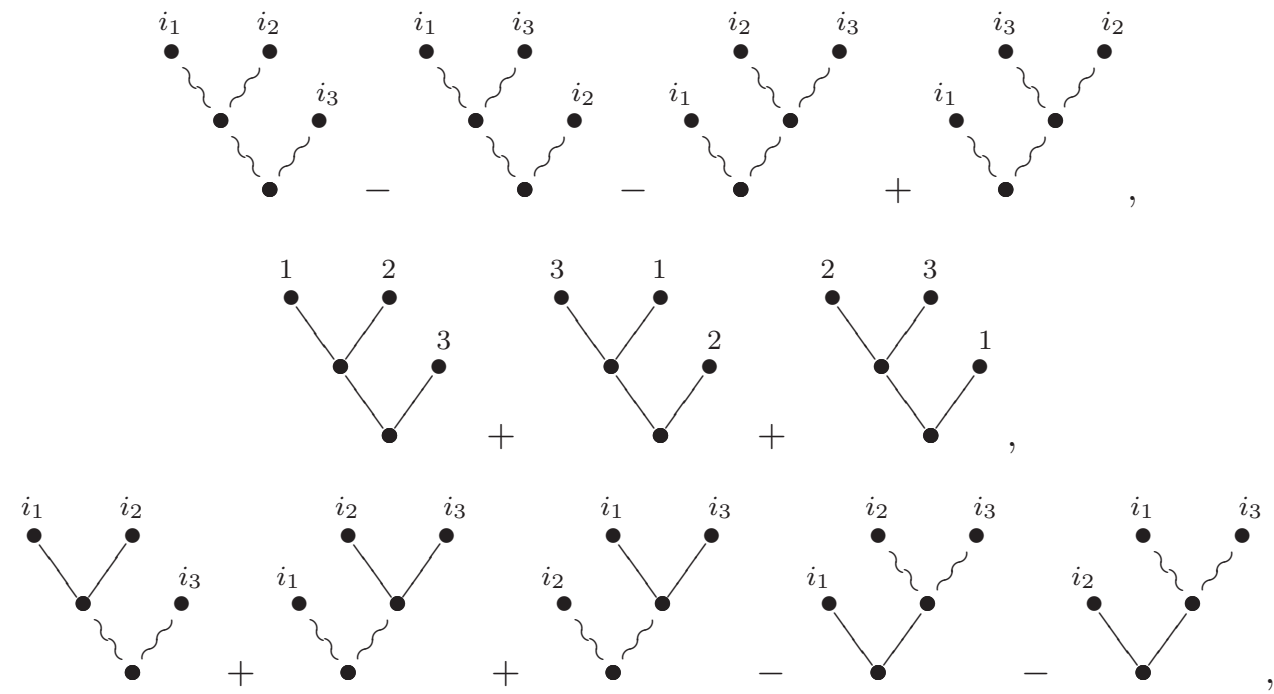

where $\left(i_{1}, i_{2}, i_{3}\right)$ is an arbitrary permutation of $(1,2,3)$.

\subsection{Koszul dual of $\mathcal{P}$}

By definition,

$$
\check{\mathcal{E}}(2)=\mathcal{E}(2)^{*} \otimes \operatorname{sgn}_{2}=k\left[\mathbb{S}_{2}\right][0] \oplus \operatorname{sgn}_{2}[1] .
$$




\section{S. A. Merkulov}

If we represent the basis vectors of $\check{\mathcal{E}}(2)$ by two planar corollas of degree zero,

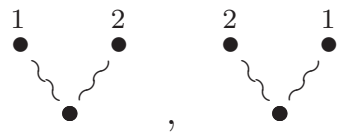

and one corolla of degree -1 ,

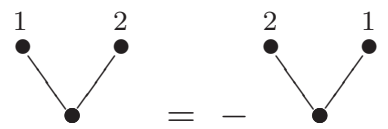

then the Koszul dual operad $\mathcal{P}^{!}$is spanned by all possible binary trees on these corollas, modulo the following relations, i.e. generators of $R^{\perp} \subset \operatorname{Free}(\hat{\mathcal{E}})(3)$,
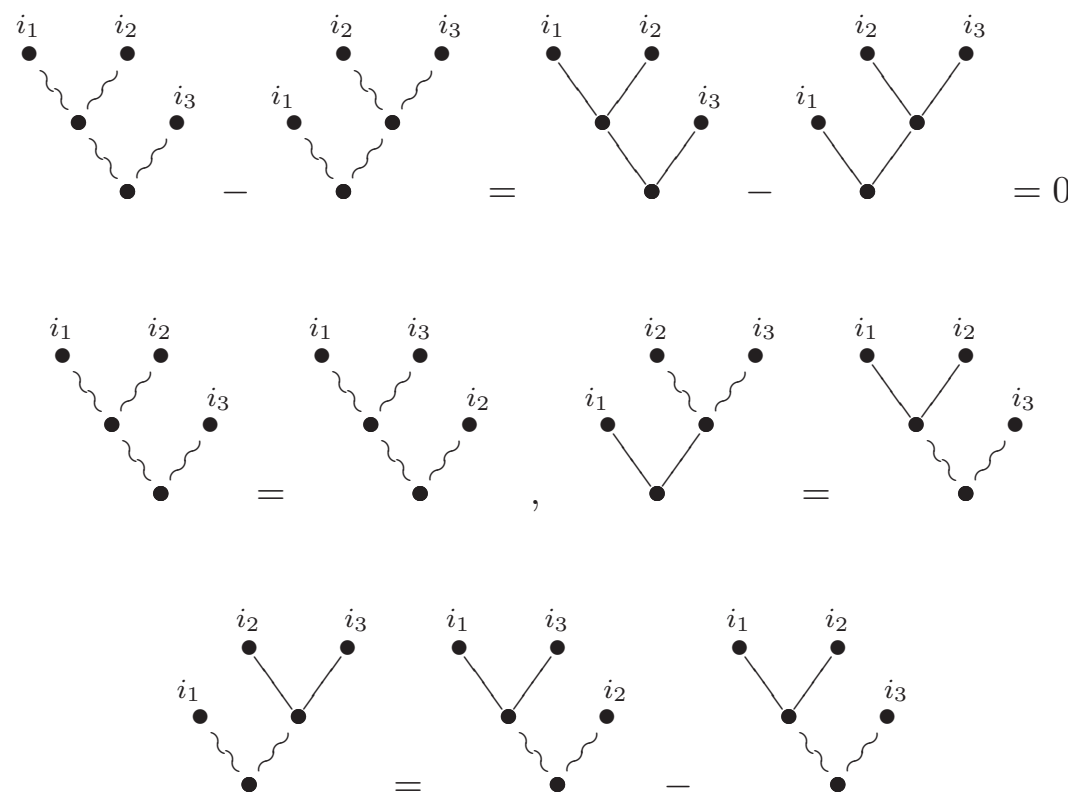

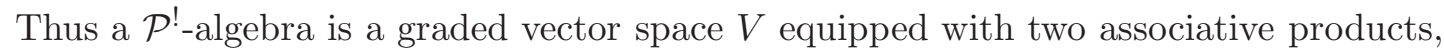

$$
\begin{aligned}
& \circ: V \otimes V \longrightarrow V \quad \bullet: \wedge^{2} V \longrightarrow V[-1] \\
& a \otimes b \quad \longrightarrow a \circ b, \quad a \otimes b \longrightarrow a \bullet b
\end{aligned}
$$

such that

$$
\begin{aligned}
& (a \circ b) \circ c=(-1)^{|b||c|}(a \circ c) \circ b, \quad a \bullet(b \circ c)=(a \bullet b) \circ c, \\
& a \circ(b \bullet c)=(-1)^{|b|+1}(a \bullet b) \circ c+(-1)^{|b|(|c|+1)}(a \bullet c) \circ b,
\end{aligned}
$$

for any $a, b, c \in V$.

Example 3.4.1. Let $(A, \cdot, d)$ be an arbitrary differential graded commutative associative algebra (say, the de Rham algebra of a smooth manifold, cf. Example 3.1.4). Then $A[-1]$ has the natural structure of a $\mathcal{P}^{!}$-algebra. If we denote elements of $A[-1]$ by $\Pi \alpha$, where $\alpha$ is an element of $A$ and $\Pi$ a formal symbol of degree 1 , then the two products in $A[-1]$,

$$
\begin{gathered}
\Pi \alpha \circ \Pi \beta=\Pi(\alpha \cdot d \beta), \\
\Pi \alpha \bullet \Pi \beta=\Pi(\alpha \cdot \beta),
\end{gathered}
$$

are associative and satisfy all the necessary conditions of $\S 3.4$. 


\section{NiJENHUIS INFINITY AND CONTRACTIBLE DIFFERENTIAL GRADED MANIFOLDS}

\subsection{Homology of pre-Lie ${ }^{2}$ algebras}

Let $\mathcal{O}$ be a quadratic operad and $V$ an $\mathcal{O}$-algebra. The $\mathcal{O}$-algebra structure on $V$ gives rise to a degree -1 differential $d_{\mathcal{O}}$ on the following positively graded vector space [GK94, MSS02],

$$
C_{\mathcal{O}} V=\oplus_{n \geqslant 1} C_{\mathcal{O}}^{n}(V)=\oplus_{n \geqslant 1}\left(\mathcal{O}^{!}(n)\right)^{*} \otimes_{\mathbb{S}_{n}}(V[1])^{\otimes n} .
$$

The associated homology, $H_{\bullet}(V):=H_{\bullet}\left(C_{\mathcal{O}} V, d_{\mathcal{O}}\right)$, is called the $\mathcal{O}$-algebra homology of $V$.

For example, if $\mathcal{O}$ is the operad of Lie algebras and $\mathfrak{g}$ a Lie algebra, then the operadic complex $\left(C_{\text {Lie }}(\mathfrak{g}), d_{\text {Lie }}\right)$ is precisely the Chevalley-Eilenberg complex,

$$
\left(\odot \cdot(\mathfrak{g}[1])=\oplus_{n}\left(\wedge^{n} \mathfrak{g}\right)[n], d_{\mathrm{CE}}\right)
$$

which computes the homology of the Lie algebra $\mathfrak{g}$ with coefficients in the trivial module.

We shall show now that the same Chevalley-Eilenberg construction can be used to compute the pre-Lie ${ }^{2}$-algebra homology.

Let $\mathcal{P}$ be the operad of pre-Lie ${ }^{2}$ algebras, and let $V$ be a $\mathcal{P}$-algebra. According to interpretation II of $\S 3.1 .2$, the vector space $V \oplus V[-1]$ is naturally a Lie algebra so that the vector space

$$
\odot^{\bullet}((V \oplus V[-1])[1])=\odot \bullet(V[1] \oplus V)
$$

has the Chevalley-Eilenberg differential $d_{\mathrm{CE}}$.

The subspace $V \subset V \oplus V[-1]$ is a Lie subalgebra with the bracket $[a, b]=a \circ b-(-1)^{|a||b|} b \circ a$. Hence we get one more Chevalley-Eilenberg complex, $\left(\odot \cdot(V[1]), d_{\mathrm{CE}}\right)$, which is a subcomplex of the one above. We define the quotient complex by the exact sequence,

$$
0 \longrightarrow\left(\odot \bullet(V[1]), d_{\mathrm{CE}}\right) \longrightarrow\left(\odot \bullet(V[1] \oplus V), d_{\mathrm{CE}}\right) \longrightarrow\left(J^{\bullet}, d\right) .
$$

Proposition 3.5.1. The quotient complex $\left(J^{\bullet}, d\right)$ is isomorphic to the operadic complex $\left(C_{\mathcal{P}}^{\bullet} V, d_{\mathcal{P}}\right)$.

The proof is a straightforward calculation.

For example, if $V$ is the pre-Lie ${ }^{2}$ algebra associated with the free Lie algebra Free $(W \oplus W[-1])$ as in Example 3.1.3, then $H_{1}(V)=W$ and $H_{2}(V)=\operatorname{Im} Q /[\operatorname{Im} Q, \operatorname{Im} Q] \neq 0$.

\subsection{Cobar construction}

Let $\mathcal{P}$ be the operad of pre-Lie ${ }^{2}$ algebras. The associated cobar construction, $\mathcal{P}_{\infty}=\mathbf{D} \mathcal{P}^{!}$, is the free operad on the $\mathbb{S}$-module,

$$
\mathcal{P}^{!}(n)^{*} \otimes \operatorname{sgn}_{n}[n-2]=\bigoplus_{p=0}^{n-1} \operatorname{Ind}_{\mathbb{S}_{n-p} \times \mathbb{S}_{p}}^{\mathbb{S}_{n-p}} \otimes \operatorname{sgn}_{p}[p-1] .
$$

Let us identify the basis of the $\left(\begin{array}{l}n \\ p\end{array}\right)$-dimensional summand $\operatorname{Ind}_{\mathbb{S}_{n-p} \times \mathbb{S}_{p}}^{\mathbb{S}_{n}} \mathbf{1}_{n-p} \otimes \operatorname{sgn}_{p}[p-1]$ with planar $[n]$-corollas of the form

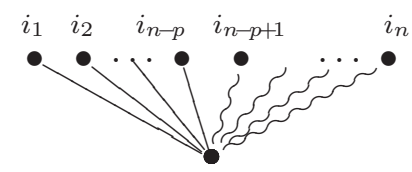

which are symmetric over the first $n-p$ inputs and antisymmetric over the last $p$ inputs, and has degree $1-p$. As an $\mathbb{S}$-module the operad $\mathcal{P}_{\infty}$ is the linear span of all possible (isomorphism classes of) plane trees formed by these corollas. The compositions $\circ_{i}^{n, n^{\prime}}$ in $\mathcal{P}_{\infty}$ are given simply by gluing the root vertex of an $\left[n^{\prime}\right]$-tree to the $i$ th tail vertex of an $[n]$-tree. To complete the picture we need just the cobar differential. 


\section{S. A. Merkulov}

Proposition 3.6.1. The cobar differential in $\mathcal{P}_{\infty}$ is given on generators by

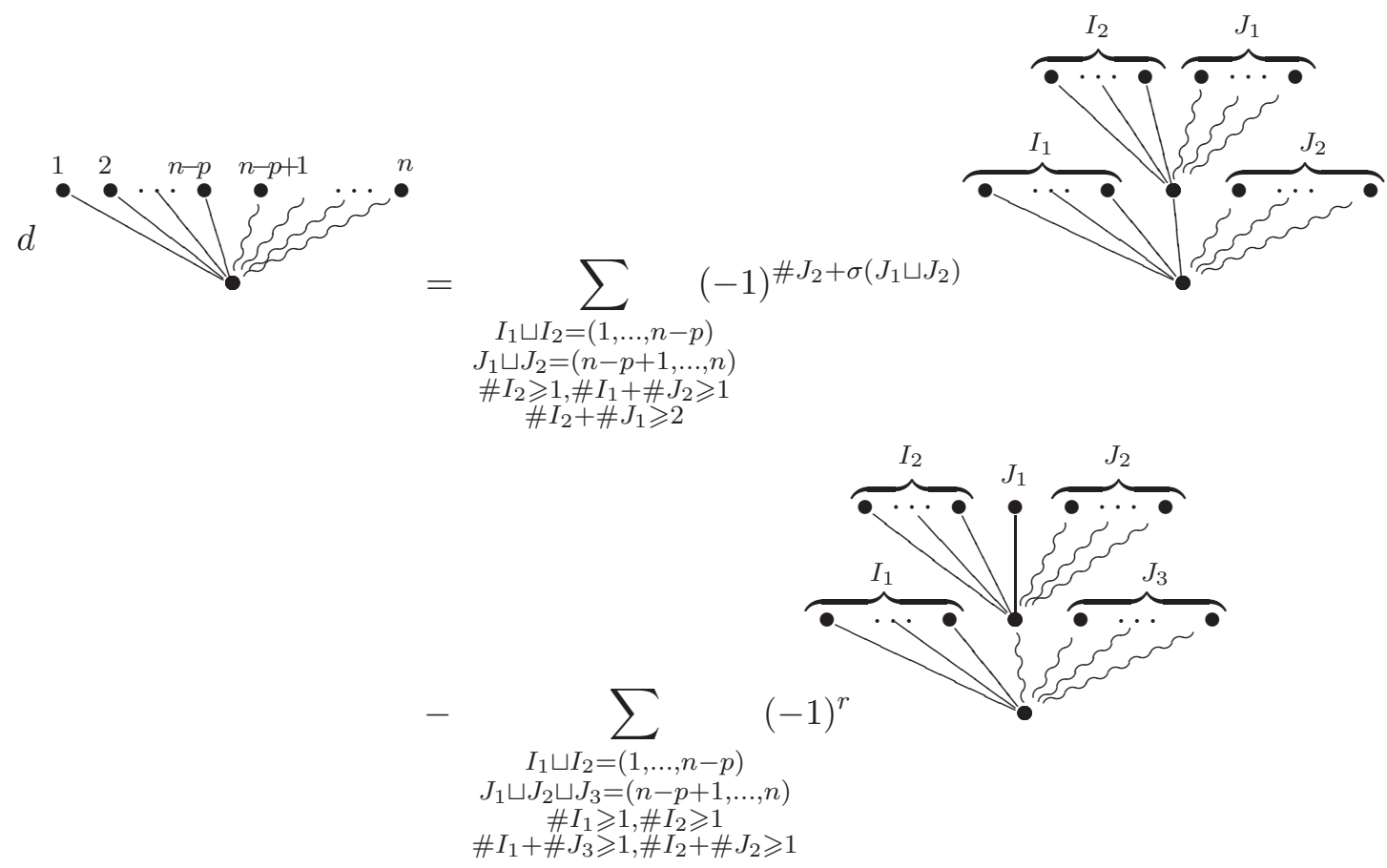

where $r=\# J_{2}+\# J_{3}+\sigma\left(J_{1} \sqcup J_{2} \sqcup J_{3}\right)$, and $\sigma\left(J_{1} \sqcup J_{2}\right)$ and $\sigma\left(J_{1} \sqcup J_{2} \sqcup J_{3}\right)$ stand for the parities of the permutations $(n-p+1, \ldots, n) \rightarrow\left(J_{1} \sqcup J_{2}\right)$ and $(n-p+1, \ldots, n) \rightarrow\left(J_{1} \sqcup J_{2} \sqcup J_{3}\right)$, respectively.

The proof is straightforward.

\subsection{Geometric interpretation of $\mathcal{P}_{\infty}$-algebras}

A pre-Lie $\infty_{\infty}^{2}$-structure on a dg vector space $V$ is a collection of linear maps,

$$
\mu_{k, p}: \odot{ }^{k} V \otimes \wedge^{p} V \rightarrow V[1-p], \quad k \geqslant 1, p \geqslant 0,
$$

satisfying a system of quadratic equations which can be read off from the equations in Proposition 3.6.1 defining the cobar differential. There is a nice geometric way to describe these equations.

Let $\hat{V}$ be the smooth formal graded manifold isomorphic to the formal neighbourhood of 0 in $V$, let $T_{\hat{V}}$ be the space of smooth vector fields, and let $\Omega_{\hat{V}}^{\bullet}$ be the de Rham algebra on $\hat{V}$.

If $\left\{e_{\alpha}, \alpha=1,2, \ldots\right\}$ is a homogeneous basis of $V$, then the associated dual basis $t^{\alpha},\left|t^{\alpha}\right|=-\left|e_{\alpha}\right|$, defines a coordinate system on $\hat{V}$. The collection of linear maps $\left\{\mu_{k, 0}\right\}_{k \geqslant 1}$ can be assembled into a germ, $\check{\partial} \in T_{\hat{V}}$, of a degree 1 smooth vector field,

$$
\text { ð }:=\sum_{k=1}^{\infty} \frac{1}{k !}(-1)^{\epsilon} t^{\alpha_{1}} \cdots t^{\alpha_{k}} \mu_{\alpha_{1} \ldots \alpha_{k}}^{\beta} \frac{\partial}{\partial t^{\beta}}
$$

where

$$
\epsilon=\sum_{i=1}^{k}\left|e_{\alpha_{i}}\right|\left(1+\sum_{j=1}^{i}\left|e_{\alpha_{j}}\right|\right)
$$

and the numbers $\mu_{\alpha_{1} \ldots \alpha_{k}}^{\beta}$ are defined by

$$
\mu_{k, 0}\left(e_{\alpha_{1}}, \ldots, e_{\alpha_{k}}\right)=\sum \mu_{\alpha_{1} \ldots \alpha_{k}}^{\beta} e_{\beta} .
$$

Here and throughout the paper we assume summation over repeated small Greek indices. 


\section{NiJENHUIS INFINITY AND CONTRACTIBLE DIFFERENTIAL GRADED MANIFOLDS}

For fixed $p \geqslant 1$, the collection of linear maps, $\left\{\mu_{k, p}\right\}_{k \geqslant 1}$, can be assembled into a germ, $\Gamma_{p} \in$ $T_{\hat{V}} \otimes \Omega_{\hat{V}}^{p},\left|\Gamma_{p}\right|=1$, of a smooth tangent vector valued differential $p$-form on $\hat{V}$,

$$
\Gamma_{p}:=\sum_{k=1}^{\infty} \frac{1}{k ! p !}(-1)^{\epsilon} t^{\alpha_{1}} \cdots t^{\alpha_{k}} \mu_{\alpha_{1} \ldots \alpha_{k}, \beta_{1} \ldots \beta_{p}}^{\gamma} \frac{\partial}{\partial t^{\gamma}} \otimes d t^{\beta_{1}} \wedge \cdots \wedge d t^{\beta_{p}}
$$

where

$$
\epsilon=\sum_{i=1}^{k}\left|e_{\alpha_{i}}\right|\left(2-p+\sum_{j=1}^{i}\left|e_{\alpha_{j}}\right|\right)+\sum_{i=1}^{k}\left(\left|e_{\beta_{i}}\right|+1\right) \sum_{j=i+1}^{k}\left|e_{\beta_{j}}\right|
$$

and the numbers $\mu_{\alpha_{1} \ldots \alpha_{k}, \beta_{1} \ldots \beta_{p}}^{\gamma}$ are defined by

$$
\mu_{k, p}\left(e_{\alpha_{1}}, \ldots, e_{\alpha_{k}}, e_{\beta_{1}}, \ldots, e_{\beta_{p}}\right)=\sum_{\gamma} \mu_{\alpha_{1} \ldots \alpha_{k}, \beta_{1} \ldots \beta_{p}}^{\gamma} \frac{\partial}{\partial t^{\gamma}} .
$$

Proposition 3.7.1. A collection of linear maps, $\left\{\mu_{k, p}: \odot{ }^{k} V \otimes \wedge^{p} V \rightarrow V[1-p]\right\}_{k \geqslant 1, p \geqslant 0}$, defines a structure of pre-Lie $e_{\infty}^{2}$-algebra on $V$ if and only if the associated degree one smooth vector field $\partial$ and the tangent vector valued differential form

$$
\Gamma:=\sum_{p \geqslant 1} \Gamma_{p} \in T_{\hat{V}} \otimes \Omega_{\hat{V}}^{\bullet}
$$

satisfy the equations

$$
[ð, \precsim]=0
$$

and

$$
\operatorname{Lie}_{\supset} \Gamma+\frac{1}{2}[\Gamma \bullet \Gamma]=0,
$$

where Lieə stands for the Lie derivative along the vector field $\precsim$ and [ $\bullet$ ] for the Frölicher-Nijenhuis brackets.

In particular, if $V$ is finite dimensional and concentrated in degree zero, then the only nonzero summand in $\Gamma$ is $\Gamma_{1} \in T_{\hat{V}} \otimes \Omega_{\hat{V}}^{1}$. According to Proposition 3.7.1, this endomorphism of the tangent bundle $T_{\hat{V}}$ makes $V$ into a pre-Lie $\infty_{\infty}^{2}$-algebra if and only if the associated Nijenhuis tensor $N_{\Gamma_{1}}=\left[\Gamma_{1} \bullet \Gamma_{1}\right]$ vanishes.

\section{Further enlargement}

From the geometric viewpoint there a small imperfection in the structure of pre-Lie $\infty_{\infty}^{2}$-algebras: the associated section $\Gamma$ of $T_{\hat{V}} \otimes \Omega_{\hat{V}}^{\bullet}$ is required to be of order at least 1 in the coordinate $t^{\alpha}$ so that constant sections are excluded. In fact, the sections $\Gamma$ which are precisely linear in $t^{\alpha}$ together with $\varpi=0$ are in one-to-one correspondence with pre-Lie ${ }_{\infty}$ structures on $V$, cf. [CL01].

It is not a problem to fix this imperfection via a further enlargement of the notion of pre-Lie algebra.

\subsection{Homologically trivial dg Lie algebras}

Such dg Lie algebras, $(\mathfrak{g}, d)$, fit into an exact sequence

$$
0 \longrightarrow \operatorname{ker} d \longrightarrow \mathfrak{g} \stackrel{d}{\longrightarrow} \operatorname{Im} d \longrightarrow 0,
$$

or, denoting $V=\operatorname{ker} d[1]$, into the exact sequence

$$
0 \longrightarrow V[-1] \longrightarrow \mathfrak{g} \longrightarrow V \longrightarrow 0,
$$

which has all arrows of degree zero. 


\section{S. A. Merkulov}

Definition 4.1.1. A Nijenhuis algebra (an $N$-algebra) is a homologically trivial dg Lie algebra, $(\mathfrak{g},[], d$,$) , together with a fixed splitting, \mathfrak{g}=V \oplus V[-1]$, of the extension (8).

Definition 4.1.2. An $N$-algebra is a graded vector space $V$ together with three binary operations,

$$
\begin{aligned}
& \circ: V \otimes V \longrightarrow V \quad[\bullet]: \odot^{2} V \longrightarrow V[1] \quad \star \quad \longrightarrow \quad \wedge^{2} V \longrightarrow \quad \longrightarrow \quad V[-1] \\
& a \otimes b \quad \longrightarrow a \circ b, \quad a \otimes b \longrightarrow(-1)^{|b|}[a \bullet b], \quad a \otimes b \longrightarrow(-1)^{|b|} a \star b
\end{aligned}
$$

such that identities (1) and (2) of Definition 3.1 as well as

$$
\begin{aligned}
&(a \circ b) \circ c-(-1)^{|b||c|}(a \circ b) \circ c-a \circ(b \circ c)+(-1)^{|b||c|} a \circ(c \circ b) \\
&=(-1)^{|b|}[a \bullet(b \star c)]-(-1)^{|b|}[a \bullet b] \star c+(-1)^{|b||c|+|c|}[a \bullet c] \star b, \\
&\left(1+\sigma+\sigma^{2}\right)\left\{(a \circ b) \star c-(-1)^{|a||b|}(b \circ a) \star c+(-1)^{|b|}(a \star b) \circ c\right\}=0
\end{aligned}
$$

hold for any $a, b, c \in V$. Here $\sigma$ stands for the cyclic permutation of the letters in the word $a b c$.

Proposition 4.1.3. Definitions 4.1.1 and 4.1.2 are equivalent.

Proof. A binary operation

$$
[,]: \wedge^{2}(V \oplus V[-1]) \longrightarrow V \oplus V[-1]
$$

satisfies the Leibnitz rule with respect to the differential

$$
\begin{array}{ccc}
d: V \oplus V[-1] & \longrightarrow & V \oplus V[-1] \\
a+\Pi b & \longrightarrow & 0+\Pi a
\end{array}
$$

if and only if it is of the form (cf. $\S 3.1 .2)$,

$$
\begin{aligned}
{[a, b] } & :=a \circ b-(-1)^{|a||b|} b \circ a+(-1)^{|a|} \Pi a \star b, \\
{[\Pi a, b]: } & =-(-1)^{|a|}[a \bullet b]+\Pi a \circ b, \\
{[\Pi a, \Pi b] } & :=\Pi[a \bullet b],
\end{aligned}
$$

for some linear maps $\circ: V \otimes V \rightarrow V,[\bullet]: \odot^{2} V \rightarrow V[1]$ and $\star: \wedge^{2} V \rightarrow V[-1]$. Then the Jacobi identities for $[$,$] are transformed into the identities of Definition 4.1.2.$

4.1.4 SUSY transformations. The set of all splittings of extension (8) is a principal homogeneous space over the Abelian group $V \otimes V^{*}[-1]$. Hence the latter acts as a kind of supersymmetry transformation on $N$-algebra structures: if $(V,[\bullet], \circ, \star)$ is an $N$ algebra, then, for any $f \in V \otimes V^{*}[-1]$, the data $\left(V,\left[\bullet_{f}\right], \circ_{f}, \star_{f}\right)$ is again an $N$-algebra, where

$$
\begin{aligned}
& {[a \bullet f b]:=[a \bullet b],} \\
& a \circ_{f} b:=a \circ b+[a \bullet f(b)]+(-1)^{|a|} f([a \bullet b]), \\
& a \star_{f} b:=a \star b+(-1)^{|a|}\{f(a) \circ b-f(a \circ b)\}-(-1)^{(|a|+1)|b|}\{f(b) \circ a-f(b \circ a)\} \\
& +(-1)^{|a|}[f(a) \bullet f(b)]-f[f(a) \bullet b]-(-1)^{|a|} f([a \bullet f(b)]),
\end{aligned}
$$

for any $a, b, c \in V$.

The category of $N$-algebras with morphisms enlarged to include supersymmetry transformations is equivalent to the category of homologically trivial dg Lie algebras. 


\section{NIJENHUIS INFINITY AND CONTRACTIBLE DIFFERENTIAL GRADED MANIFOLDS}

\subsection{Operad of $N$-algebras}

Let us denote this operad by $N$. It is quadratic, $N=\operatorname{Free}(\mathcal{E}) /\langle R\rangle$, with $\mathcal{E}(2):=k\left[\mathbb{S}_{2}\right][0] \oplus \mathbf{1}_{2}[-1] \oplus$ $\operatorname{sgn}_{2}[1]$. Let the corollas

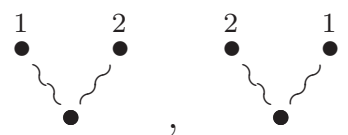

stand for the basis of $k\left[\mathbb{S}_{2}\right][0]$ and the corollas

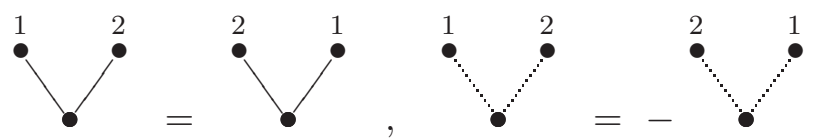

for the basis vectors of the one-dimensional representations $1_{2}[-1]$ and $\operatorname{sgn}_{2}[1]$, respectively. Then the ideal $\langle R\rangle$ is generated by (3) and (4) as well as the following elements of Free $(\mathcal{E})(3)$,

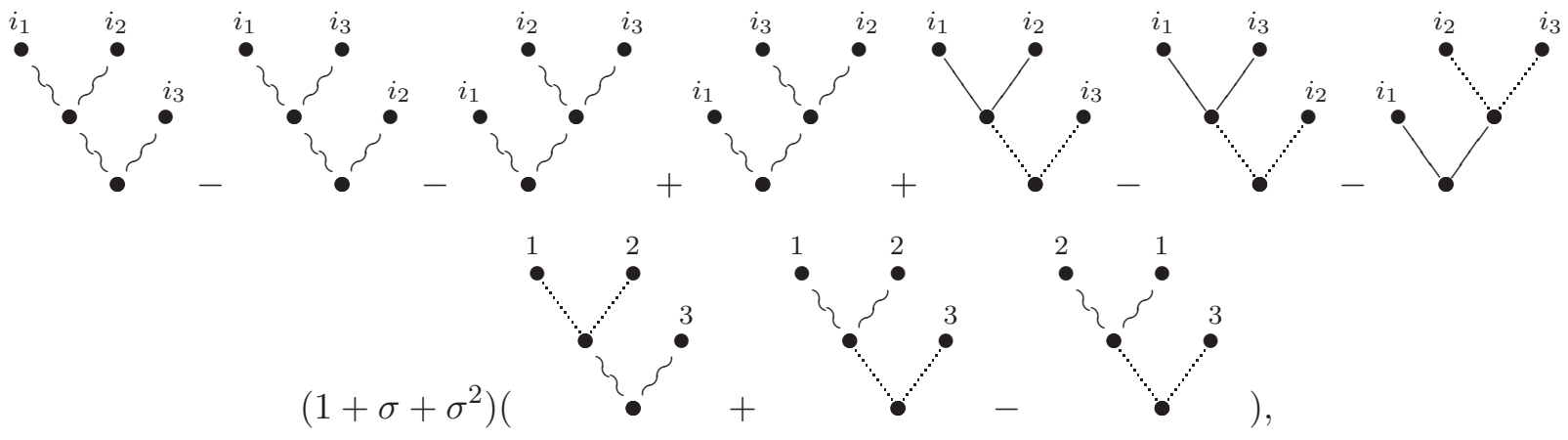

where $\sigma$ is the cyclic permutation of $(1,2,3)$.

\subsection{Koszul dual of $N$}

By definition, $N^{!}=\operatorname{Free}(\check{\mathcal{E}}) /\left\langle R^{\perp}\right\rangle$, with the only non-vanishing bit of the $\mathbb{S}$-module $\check{\mathcal{E}}$ being $\check{\mathcal{E}}(2):=$ $k\left[\mathbb{S}_{2}\right][0] \oplus \operatorname{sgn}_{2}[1] \oplus \mathbf{1}_{2}[-1]$. Let the corollas

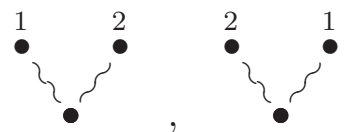

stand for the basis of $k\left[\mathbb{S}_{2}\right][0]$ while the corollas

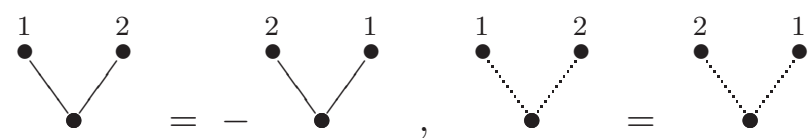

represent the basis vectors of $\operatorname{sgn}_{2}[1]$ and $\mathbf{1}_{2}[-1]$, respectively. Then the ideal $\left\langle R^{\perp}\right\rangle$ is generated by $(5)-(7)$ and following elements of $\operatorname{Free}(\breve{\mathcal{E}})(3)$,

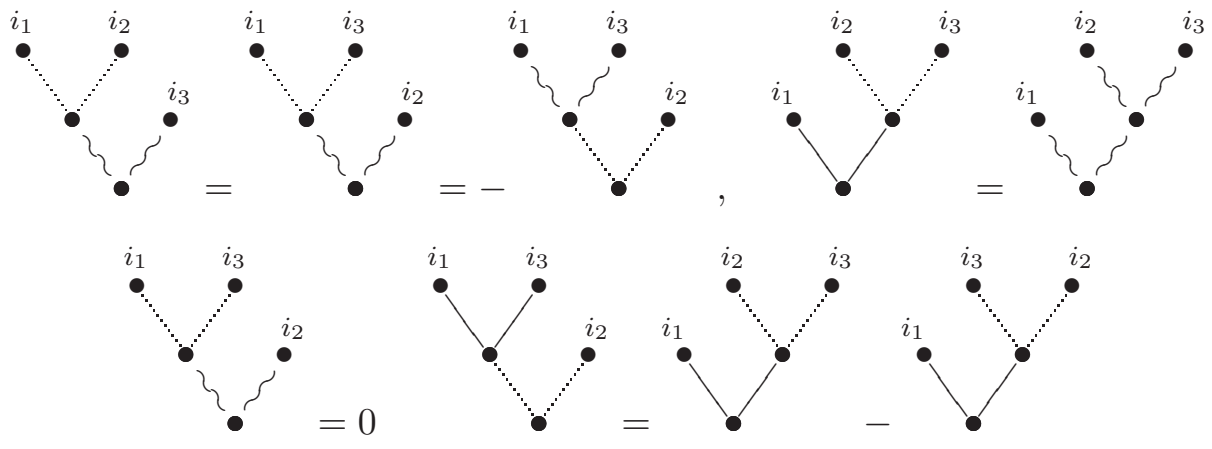




\section{S. A. Merkulov}

Example 4.3.1. If $(A, \cdot, d)$ is a differential graded commutative associative algebra, then $A[-1]$ has a natural structure of $N^{!}$-algebra with the products (cf. Example 3.4.1)

$$
\begin{aligned}
& \Pi \alpha \circ \Pi \beta=\Pi(\alpha \cdot d \beta), \\
& \Pi \alpha \bullet \Pi \beta=\Pi(\alpha \cdot \beta), \\
& \Pi \alpha \star \Pi \beta=(-1)^{|a|} \Pi(d \alpha \cdot d \beta),
\end{aligned}
$$

where $\circ, \bullet$ and $\star$ correspond, respectively, to the generators

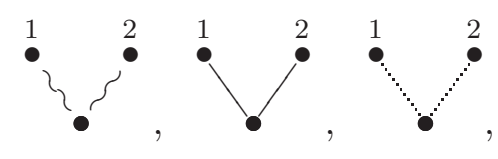

of the operad $N$ !

\section{Geometric description of Nijenhuis N $_{\infty}$ algebras}

The cobar construction, $N_{\infty}:=\mathbf{D} J^{!}$, is the free operad on the $\mathbb{S}$-module $\bigoplus_{p=0}^{n} \operatorname{Ind}_{\mathbb{S}_{n-p} \times \mathbb{S}_{p}}^{\mathbb{1}_{n-p}} \otimes$ $\operatorname{sgn}_{p}[p-1]$. The difference from the pre-Lie ${ }^{2}$ case in $\S 3.6$ is an extra summand corresponding to $p=n$. The cobar differential has the form of Proposition 3.6.1 with the summation ranges extended to include:

- the case $\# I_{2}=0$ in the first sum; and

- the case $\# I_{1}=0$ in the second sum.

Hence an $N_{\infty}$-structure on a graded vector space $V$ can be described as

- a collection of linear maps, $\left\{\mu_{k, p}: \odot^{k} V \otimes \wedge^{p} V \rightarrow V[1-p]\right\}_{k \geqslant 1, p \geqslant 0}$, exactly the same as in the case of pre-Lie ${ }^{2}$ algebras; plus

- an additional collection of linear maps $\left\{\mu_{0, p}: \wedge^{p} V \rightarrow V[1-p]\right\}_{p \geqslant 1}$,

satisfying the equations which can be interpreted as in Proposition 3.7.1: the differential form

$$
\Gamma:=\sum_{p \geqslant 1} \sum_{k=0}^{\infty} \frac{1}{k ! p !}(-1)^{\epsilon} t^{\alpha_{1}} \cdots t^{\alpha_{k}} \mu_{\alpha_{1} \ldots \alpha_{k}, \beta_{1} \ldots \beta_{p}}^{\gamma} \frac{\partial}{\partial t^{\gamma}} \otimes d t^{\beta_{1}} \wedge \cdots \wedge d t^{\beta_{p}} \in T_{\hat{V}} \otimes \Omega_{\hat{V}}^{\bullet}
$$

satisfies $\operatorname{Lie}_{ð} \Gamma+\frac{1}{2}[\Gamma \bullet \Gamma]=0$. Representations of $N_{\infty}$ in vector spaces concentrated in degree zero are obviously in one-to-one correspondence with formal germs of Nijenhuis geometric structures which vanish at 0 .

There is, however, another more coherent picture of $N_{\infty}$ algebras.

\subsection{Reminder on the homotopy classification of $L_{\infty}$ algebras [Kon03]}

Let $L$ be an operad of Lie algebras and $L_{\infty}$ its cobar construction (which happens to be the minimal resolution of $L$ as the latter is Koszul). There is a one-to-one correspondence between $L_{\infty}$ algebra structures on a dg vector space $V$ and degree one vector fields $\partial$ on $\hat{V}$, the formal neighbourhood of zero in $V$, which vanish at 0 and satisfy the integrability condition $[ð, ð]=0$. The data $(\hat{V}, ð)$ is often called a smooth formal dg manifold.

Hence the homotopy theory of $L_{\infty}$ algebras is the same as the homotopy theory of formal dg manifolds.

An $L_{\infty}$ algebra $(\hat{V}, \partial)$ is said to be minimal if the first, $\partial_{1}$, Taylor coefficient of the homological vector field $\partial$ vanishes. It is said to be linear contractible if the higher Taylor coefficients, $\partial_{\geqslant 2}$, vanish and the first one, $\check{\partial}_{1}$, has trivial cohomology when viewed as a differential in $V$. 


\section{NiJENHUIS INFINITY AND CONTRACTIBLE DIFFERENTIAL GRADED MANIFOLDS}

According to Kontsevich [Kon03], any dg manifold is $L_{\infty}$-isomorphic to the direct product of a minimal and a linear contractible dg manifold. A dg manifold is said to be contractible if it is $L_{\infty}$-isomorphic to a linear contractible dg manifold.

\subsection{Nijenhuis $_{\infty}$ versus contractible $\mathrm{dg}$ manifolds}

Let $\mathcal{M}$ be a formal neighbourhood of zero in $V \oplus V[-1]$. The standard differential $d$ on $V \oplus V[-1]$ induces a homological vector field on $\mathcal{M}$ which we denote by the same letter. Thus $(\mathcal{M}, d)$ is a linear contractible dg manifold.

Let $i: T_{\hat{V}} \otimes \Omega_{\hat{V}}^{\bullet} \rightarrow T_{\mathcal{M}}$ be degree -1 linear map of Example 3.1.4 which sends sections of $T_{\hat{V}} \otimes \Omega_{\hat{V}}^{\bullet}$ into vertical vector fields on the fibration $\mathcal{M} \rightarrow \hat{V}$. Consider the degree zero map

$$
\begin{aligned}
\Psi: T_{\hat{V}} \otimes \Omega_{\hat{V}}^{\bullet} & \longrightarrow c \\
X & \longrightarrow[d, i(X)] .
\end{aligned}
$$

The equation Lie $_{\partial} \Gamma+\frac{1}{2}[\Gamma \bullet \Gamma]=0$ implies that $\hat{\partial}=\Psi(\partial+\Gamma)$ is a homological vector field on $\mathcal{M}$ which commutes with $d$. Thus we obtain the following statement.

TheOREM 5.2.1. There is a one-to-one correspondence between Nijenhuis $s_{\infty}$ structures in a dg vector space $V$ and homological vector fields on $\mathcal{M}$ which commute with $d$.

A Nijenhuis $_{\infty}$ algebra is said to be minimal if the homological vector field $\check{\partial}$ (or, equivalently, $\hat{\partial}$ ) is minimal, i.e. its first Taylor coefficient vanishes. A more invariant way to formulate this condition is to say that $\hat{\partial} I \subset I^{2}$, where $I$ is the ideal of the distinguished point $0 \in \mathcal{M}$. Then it follows from Theorem 5.2.1 that $d+\hat{\partial}$ is a homological vector field on $\mathcal{M}$ whose first Taylor coefficient coincides with $d$.

COROLlary 5.2.2. There is a one-to-one correspondence

$$
\{\text { minimal Nijenhuis } \infty \text { algebras }\} \stackrel{1: 1}{\longleftrightarrow}\{\text { contractible } d g \text { manifolds }\} .
$$

As the operad $N_{\infty}$ is cofibrant, any Nijenhuis $\infty_{\infty}$ algebra is homotopy equivalent to a minimal one. Hence we have the following.

Corollary 5.2.3. Any Nijenhuis $\infty$ algebra is homotopically equivalent to a contractible $d g$ manifold.

\section{ACKNOWLEDGEMENT}

The author was partially supported by the Göran Gustafsson foundation.

\section{REFERENCES}

GJ94 E. Getzler and J. D. S. Jones, Operads, homotopy algebra, and iterated integrals for double loop spaces, Preprint (1994), hep-th/9403055.

GK94 V. Ginzburg and M. Kapranov, Koszul duality for operads, Duke Math. J. 76 (1994), 203-272.

CL01 F. Chapoton and M. Livernet, Pre-Lie algebras and the rooted trees operad, Internat. Math. Res. Notices 8 (2001), 395-408.

HM99 C. Hertling and Yu. I. Manin, Weak Frobenius manifolds, Internat. Math. Res. Notices 6 (1999), $277-286$.

Kon03 M. Kontsevich, Deformation quantization of Poisson manifolds, Lett. Math. Phys. 66 (2003), $157-216$. 


\section{NiJENHUIS INFINITY AND CONTRACTIBLE DIFFERENTIAL GRADED MANIFOLDS}

MSS02 M. Markl, S. Shnider and J. D. Stasheff, Operads in algebra, topology and physics (American Mathematical Society, Providence, RI, 2002).

Nij55 A. Nijenhuis, Jacobi-type identities for bilinear differential concomitants of certain tensor fields, Indag. Math. 17 (1955), 390-403.

Mer04a S. A. Merkulov, Operads, deformation theory and F-manifolds, in Frobenius manifolds, Aspects Math. E36 (Vieweg, Wiesbaden, 2004), 213-251.

Mer04b S. A. Merkulov, PROP profile of Poisson geometry, Preprint (2004), math.DG/0401034.

Mic87 P. W. Michor, Remarks on the Frölicher-Nijenhuis bracket, in Differential geometry and its applications, Brno, 1986 (Reidel, Dordrecht, 1987), 197-220.

Sta63 J. D. Stasheff, On the homotopy associativity of H-spaces, I, II, Trans. Amer. Math. Soc. 108 (1963), 272-292, 293-312.

S. A. Merkulov sm@math.su.se

Matematiska institutionen, Stockholm universitet, 10691 Stockholm, Sweden 